\title{
面向异构信息嵌入模型的交互对比可视分析系统
}

\author{
王似奸, 汤焂* \\ (浙江工业大学计算机科学与技术学院 杭州 310023) \\ (ytang@zjut.edu.cn)
}

\begin{abstract}
摘 要: 为理解不同异构网络嵌人模型之间的差异, 并解决评估异构网络嵌入模型所存在的定性分析复杂且隐藏的问题, 对比分析方法首先应统一模型的评估指标和任务, 然后训练模型以获取模型训练过程中的大量参数和特征信息, 并保留 完整且非均值化的评估结果进行可视化. 基于模型参数和特征数据, 设计并实现一个交互对比可视分析工具一 HINCompare, 包括基础评估指标的分布概览和推荐结果对比视图, 以及模型嵌人过程中融合的局部拓扑结构特征视图. 该工具支持探索模型中不同特征聚合方法所存在的共同模式和不同架构的模型之间的差异. 此外, HINCompare 通过热 力图展示了用户在电影类型和年份上的偏好特征, 可结合推荐结果的上下文信息进行分析和评估, 解决推荐中的黑盒问 题, 提供推荐结果的来源信息, 增加可解释性. 最后, 通过真实的豆瓣电影数据验证了系统的有效性.
\end{abstract}

\author{
关键词: 可视分析; 异构信息网络; 异构网络嵌人模型; 可解释性推荐 \\ 中图法分类号: TP391.41 DOI: 10.3724/SP.J.1089.2021.19260
}

\section{Visual Analysis and Interactive Comparison for Heterogeneous Information Network Embedding Model}

\author{
Wang Youyan and Tang Ying* \\ (College of Computer Science and Technology, Zhejiang University of Technology, Hangzhou 310023)
}

\begin{abstract}
To understand the differences between various heterogeneous information networks (HINs) embedding models, and to solve intricate and hidden problems in model evaluation, the comparative analysis method should include the following steps. First, the evaluation indicators and tasks are made consistent for all models. Then the parameters and structural features are extracted from the model training process and the complete and non-averaged evaluation results are retained for visualization. Based on the model parameters and characteristics data, we design and implement a visual analysis tool named HINCompare, which includes an overview of the distribution of basic evaluation indicators, a comparison view of recommended results, and a feature view of the local topology structure for model embedding. The tool allows developers to explore the common patterns of different feature aggregation methods of different models and the differences in their architectures. In addition, HINCompare shows user's preferences for movie types and years with heat maps, which are combined with the contextual information of the recommended results for further analysis and evaluation. The system provides insights into black-box problems of models and increases interpretability by supplying information about the sources of the results. We conduct the preliminary evaluation study with Douban movie data to verify the effectiveness of the system.
\end{abstract}

收稿日期：2021-07-06; 修回日期: 2021-08-24. 基金项目：国家自然科学基金面上项目(61972355); 浙江省公益技术研究计划 (LGG19F020012). 王做妍(1996一), 女, 硕士研究生, 主要研究方向为数据分析与可视化; 汤颖(1977一), 女, 博士, 教授, 博士生导师, CCF 会员, 论文通讯作者, 主要研究方向为大数据分析与可视化、计算机图形学. 
Key words: visual analysis; heterogeneous information network; heterogeneous network embedding model; interpretable recommendation

现实中的对象及其相互关系通常都是多类型 的, 为了在数据挖掘中更好地保留其复杂的结构信 息和丰富的语义信息, 研究人员将这些多类型的对 象和关系, 甚至来源于不同数据源的信息融合成单 个信息网络, 并称之为异构信息网络 ${ }^{[1]}$.

诚然, 当前已存在许多成功的且应用广泛的异 构网络嵌人模型, 可通过节点分类、连接预测和推 荐等任务进行评估. 这些任务结合相应的模型评估 指标, 如准确率, 可以定量地分析网络嵌人的性能. 但目前针对异构网络嵌人模型的定性分析仍然缺 失, 即嵌人结果是否保留了结构或语义信息, 尤其 在评估值相似的情况下, 如何判断不同架构的嵌人 模型存在的差异, 以及网络嵌人过程中存在的共同 模式 ${ }^{[2]}$. 同样, 理解不同嵌人模型之间的差异对于提 高模型的有效性和预测精度以及实现公平性至关重 要 ${ }^{[3]}$. 特别是在推荐任务中, 不同模型的评估值相 似, 是否意味着推荐结果的有效性一致, 同一模型 对不同用户的推荐是否保留其个性化, 这些问题对 于推荐都十分值得探索.

可视化技术作为热点研究方法, 具有赋予抽象 数据直观形象的能力, 它超越了传统的统计学评估 指标, 可以提供丰富的上下文信息, 已成功应用于 机器学习领域, 如模型理解 ${ }^{[4]}$ 、决策分析 ${ }^{[5]}$. 因此, 借助可视化的手段, 可以让用户自主地观察、探 索、挖掘和分析数据与模型、模型与模型之间的模 式, 以及直观地展示推荐结果与模型之间的关联, 从而实现对异构嵌人模型的定性分析.

为了解决上述问题, 本文设计并实现了一个模 型可视化对比分析系统.

(1) 利用豆瓣电影数据, 对 3 个具有代表性的 不同架构的异构信息网络嵌人模型 HetGNN ${ }^{[6]}$, $\mathrm{KGAT}^{[7]}$ 和 $\mathrm{NIRec}^{[8]}$ 进行训练, 统一下游任务与评估 指标, 保留推荐结果以及针对每位用户的推荐评估 结果.

（2）针对异构网络嵌人过程中融合的局部拓扑 结构和语义信息进行可视化, 分析测试实例和模型 架构之间的关联, 以便于更好地理解模型学习过程 中不同邻居特征聚合机制对最终嵌人结果的影响.

(3) 利用多个视图的交互联动, 向用户提供上 下文信息, 增加推荐结果的可解释性, 并提供快照 保存功能, 便于进行回溯和对比.

\section{1 相关工作}

\section{1 异构网络嵌入}

异构网络嵌人旨在学习将输人空间映射到保 持网络结构和语义信息的低维空间中的函数 ${ }^{[9]}$. 早 期研究将异构信息网络分解为多个同质网络再分别 进行嵌人学习. 如 Shi 等 ${ }^{[10]}$ 利用元路径从异构网络 中抽取出多个同构网络, 对其进行表示学习并融合; Chen 等 ${ }^{[11]}$ 通过一个特定的矩阵将异构网络分解为 多个基于关系的子网络进行表示学习.

近年来, 随着深度神经网络的发展, 一些研究 人员尝试将深度模型应用到异构网络嵌人中, 与上 述浅层模型相比, 深度模型使用神经网络从节点属 性和节点间的交互中学习嵌人, 能够更好地捕获非 线性关系，从而保留更多的结构信息和语义信息. 目前, 该方法大致可分为 2 类: 一是利用专家知识 设计的元路径对网络嵌人进行学习; 二是利用信息 传递机制聚合目标节点周围的邻居特征进行高阶关 系的建模. Wang 等 ${ }^{[12]}$ 基于元路径的模型提出了一 种层次化的注意力机制学习不同节点和元路径的重 要性, 同时捕获了异构网络的结构信息和语义信息. $\mathrm{Hu}$ 等 ${ }^{[13]}$ 受 Transformer 模型启发, 提出基于信息传 递机制捕获不同类型节点之间的交互的方法, 在聚 合过程中学习邻居的权重, 以此考虑不同邻居所传 递信息的重要性.

然而, 这 2 类方法在理论上有着各自的优缺点, 如元路径的方法因其需要领域知识而被认为具有一 定局限性, 信息传递机制的方法因其聚合了周围所 有邻居的特征被认为引人了噪声信息. 通过不同方 式学习的用户特征究竟有何差异? 是否有效地保留 了其特征信息? 因此, 本文保留了模型训练过程中 的路径特征信息, 通过可视化的方式对上述问题进 行更加细粒度的分析.

\section{2 模型对比可视化}

模型对比通常采用统计学的评估指标, 如准确 率均值和召回率均值等, 它们可有效地对模型参数 修改前后进行对比, 但针对不同架构的模型, 均值 可能会掩盖模型内在的一些重要模式 ${ }^{[14]}$. 例如, Ren 等 ${ }^{[15]}$ 指出, 2 个不同分类器在相同数据中产生一样 的预测精度, 但预测分数的分布情况却大相径庭, 然而, 这些分数的分布情况对于评估预测错误和调 试模型性能是非常重要的. 
可视化技术具有赋予抽象数据直观形象的能 力, 能以显式的方式提供模型的上下文信息 ${ }^{[16]}$, 使 用户能够在抽象级别上比较模型的性能, 必要时进 行详细的分析 ${ }^{[17]}$. 如, Zeng 等 ${ }^{[18]}$ 探索卷积神经网络 学习过程中不同迭代次数和不同学习参数对最终结 果的影响; Zagoruyko 等 ${ }^{[14]}$ 通过热力图展示了模型 的神经元激活状况, 并提供了模型的总体指标概览, 使其能够识别和比较错误分类的激活模式, 其局限 性在于该系统需要有明确标记的数据, 而不能应用 于没有具体对错的推荐任务中; Alexander 等 ${ }^{[19]}$ 从 主题模型的单模型使用中推导出比较任务, 结合颜 色和位置编码, 使分析人员能够轻松地查看文档相 似性的变化.

以上大多数工作都集中在分析一个单一的模 型, 且处理的数据多为可直接观察的图像数据; 而 异构网络嵌人将高维特征映射成低维向量, 无法进 行直接对比或直观理解其学习过程中所包含的特 征. 此外, 现有的多模型可视对比系统虽然可以通 过可视化神经元的激活状况分析模型内在特征与模 型表现, 但此方法并不适用于异构网络嵌人模型. 因此, 本文需要设计一个全新的可视化方法对不同 架构的异构网络嵌人模型进行对比分析, 并使模型 所学特征能与推荐结果进行综合分析与比较, 在没 有明确标签的情况下评估模型仍然是一个挑战.

针对嵌人向量无法进行直接对比的问题, 本文 将所学特征向量应用于下游推荐任务, 通过对目标 推荐结果的验证和自下而上的探索方式, 理解模型 学习过程以及最终嵌人特征. 针对现有方法无法对 异构嵌人模型进行可视化的问题, 本文保留了模型 学习过程中的特征信息和重要度系数, 并增加了更 多上下文信息和多维度相关性分数, 以便帮助用户 自主判断推荐结果的合理性和正确性.

\section{2 问题定义和数据准备}

本文选择了 3 种具有代表性的异构信息网络 嵌人模型支持工作目标.

\section{1 异构信息网络相关概念定义}

异构网络 $H$. 异构网络由不同类型的节点和边 构成, 表示为 $H=\{V, E, \phi, \varphi\}$, 每个节点 $v_{i} \in V$, 有一个 类型映射函数 $\phi\left(v_{i}\right)=A_{i}, A$ 为节点类型; 每条边 $e_{i} \in E$, 有关系映射函数 $\varphi\left(e_{i}\right)=R_{i}, R$ 表示边类型.

元路径 $L$. 元路径是在异构信息网络上定义的
路径, 以 $\stackrel{R_{0}}{\rightarrow} \stackrel{R_{1}}{\rightarrow} A_{1} \rightarrow \stackrel{R_{n}}{\rightarrow} A_{2} \rightarrow \cdots$ 的形式表示, 其中, $A$ 和 $R$ 分别代表节点类型和边类型.

以图 1 所示的电影异构网络数据为例, 其包含 5 种类型的节点, 以及用户观看电影等 8 种类型的 关系. 其中, 元路径 $U \stackrel{\text { rate }}{\rightarrow} M \stackrel{\text { direct }^{-1}}{\rightarrow} D \stackrel{\text { direct }}{\rightarrow} M$ (简记为 UMDM)表示目标电影与用户观看过的电影由相同 导演执导, $U \stackrel{\text { rate }}{\rightarrow} M \stackrel{\text { act }^{-1}}{\rightarrow} A \stackrel{\text { act }}{\rightarrow} M$ (简记为 UMAM) 表示 目标电影与用户观看过的电影由相同演员参演, 它 们包含了不同的语义信息. 此外, 电影节点包含各 类属性信息, 如标签等.

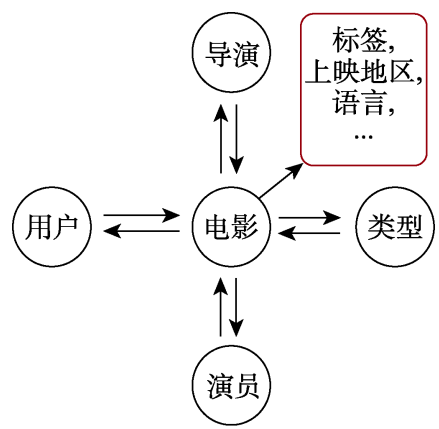

图 1 电影数据的异构信息网络结构

\section{2 异构信息嵌入模型介绍}

针对第 1.1 节提出的问题, 本文选择了 3 个具 有代表性的最新异构网络嵌人模型进行探索：(1) 基于元路径的卷积交互模型 $\mathrm{NIRec}^{[8]}$, 该模型受信 号卷积的启发, 利用卷积操作对用户和物品之间基 于元路径的交互信息进行学习, 并通过层次注意力 机制对节点级和路径级的特征信息进行聚合. (2) 基 于信息传递机制的注意力融合模型 $\mathrm{KGAT}^{[7]}$, 该模 型将图注意力网络应用于知识图与用户-物品二分 图相结合的异构信息网络中, 以图卷积的形式递归 式地挖掘高阶连通信息, 实现了高阶关系的节点表 示学习. (3) 基于随机游走的图表示学习模型 $\mathrm{HetGNN}^{[6]}$, 该模型提出了统一的框架, 同时考虑异 构网络的结构信息和节点属性信息, 并将学习过程 与下游任务分开, 以满足异构信息网络的下游任务.

\section{3 数据准备}

为了以系统且公平的方式评估嵌人模型, 并为 模型和推荐结果提供上下文信息, 本文从真实的豆 瓣电影网站中收集了 2 万份豆瓣用户数据和 2 万部 豆瓣电影数据 ${ }^{\circledR}$, 为防止出现无效数据, 以用户的观 影记录至少大于 50 条、电影被有效用户观看次数

(1) https://github.com/Wyy-96/Douban_Data 
大于 20 等条件进行了数据过滤. 又因推荐模型通常 存在冷启动问题, 为此保留 $10 \%$ 的观影记录小于 50 条的用户作为冷启动数据. 最终数据的电影被观看 人次分布情况如图 2a 所示, 用户观影历史记录分布 如图 2b 所示.

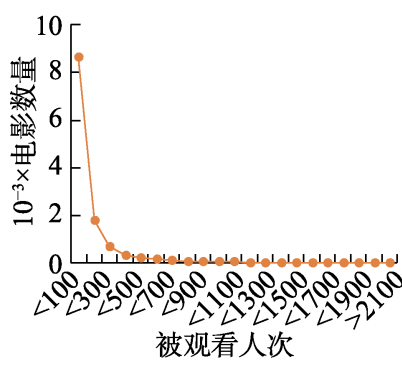

a. 电影被观看人次分布

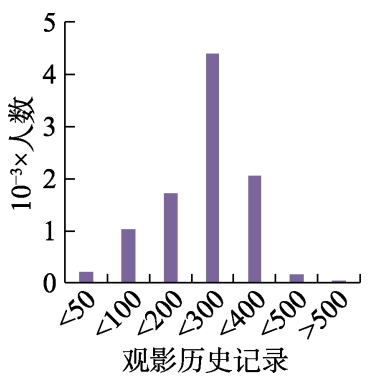

b. 用户观影历史分布
图 2 数据分布情况

\section{4 系统设计目标}

G1. 传统评估指标的联合分析. 任何模型的构 建, 必定会经过从训练、验证到与同类先进模型进 行比较和评估的过程. 在模型调优中, 研究人员利 用评估指标作为指导, 根据领域知识和直觉修改参 数和模型架构. 在模型评估中, 指标能够将模型与 大量、广泛的其他先进模型进行定量比较, 因此评 估指标是常用且不可或缺的模型对比方式.

G2. 模型之间的共同模式. 现有异构网络嵌人 模型的特征聚合方法大致分为 2 类，即基于给定元 路径聚合邻居信息, 及聚合一定范围内的所有直接 邻居信息. 那么基于元路径的邻居与一定范围内的 邻居是否有内在关联, 它们对目标节点最终的贡献 有何差异, 对最终的嵌人结果有何影响, 能否通过 某种聚合方式进行统一, 这对于当前异构网络嵌人 模型的发展十分重要 ${ }^{[2]}$.

G3. 推荐结果的细节和可解释性. 模型对比通 常使用评估指标的均值, 使开发人员无法发现预测 分数的分布情况对模型的影响, 忽略了不同个体的 差异性以及不同模型的推荐模式. 例如, 推荐结果 没有预测正确, 但其特征非常符合用户的观影偏好, 或是模型的推荐总是偏向于某种特征. 若使用可视 化工具展示推荐结果的上下文信息和推荐指标的细 节, 可以让模型评估更加深人, 甚至带来一些其他 启发.

\section{5 评估指标}

为了对模型进行公平的评估, 完成目标 G1, 本 文采用异构网络用于推荐任务的最常见评估指标, 即精确率、召回率和受试者工作特征曲线面积 (area under the curve, AUC), 对 3 个嵌人模型进行 总体评估. 另外, 为了探索模型是否保留了个性化, 增加推荐结果的相似性评估, 计算不同用户的推荐 列表之间的余弦相似性. 用户 $i$ 的个性化分数 $P$ 计 算过程为

$$
P_{i}=1-\frac{1}{|U|-1} \sum_{i^{\prime} \in U, i^{\prime} \neq i} \frac{\boldsymbol{q}_{i} \cdot \boldsymbol{q}_{i^{\prime}}}{\left\|\boldsymbol{q}_{i}\right\| \times\left\|\boldsymbol{q}_{i^{\prime}}\right\|} .
$$

其中, $U$ 表示用户集合; $\boldsymbol{q}_{i}$ 表示用户 $i$ 的某个推荐列 表所构成的向量.

\section{3 系统概述}

\section{1 设计需求}

为达到第 2.4 节中提到的系统设计目标, 本文 确定了以下 5 个设计需求指导系统设计.

R1. 提供模型评估指标的概述 (G1).

R2. 提供异构信息网络嵌人模型在聚合邻居过 程中的细节信息(G2).

R3. 识别嵌入结果对用户的推荐模式(G2).

R4. 提供推荐结果的生成来源(G3).

R5. 为推荐结果提供上下文信息(G3).

\section{2 系统流程}

要从大量用户的推荐结果出发寻找模型更加 细粒度的损失, 分析人员通常需要操作数据并选择 上下文. 为此, 本文通过自下而上的数据展开流程, 按需显示上下文信息. 如图 3 所示, 该系统由 5 个部 分组成, 数据窗口提供了模型选择功能以及数据信 息展示, 可通过开关选择任意模型加人对比. 评估 指标概览图(R1)提供了模型在 4 个评估指标下的表 现分布情况, 分析人员可根据其需求䇻选感兴趣的 数据, 再通过环形刷选择目标用户. 针对某一感兴 趣目标用户，模型细节评估图(R3)将展示模型在不 同指标下的综合表现，以及对当前目标用户推荐结 果的韦恩图. 点击韦恩图中表示推荐电影的散点, 会在推荐结果溯源图(R2, R4)中展示该推荐电影与 目标用户之间的路径信息; 点击感兴趣的节点, 可 展开查看其详细实体节点信息, 鼠标悬停将高亮显 示该节点与周围邻居的关系, 并可在右上角切换当 前面板展示的模型路径. 此外，上下文信息关联图 (R5)提供了选定推荐结果的上下文信息以及与目标 用户在类型、演员、导演、标签、相同观看人次等 5 个属性下的相关性直方图, 可与模型细节评估图中 目标用户的观影记录热力图进行联合分析, 以便评 估推荐电影在各属性上与用户偏好的关联. 点击快 


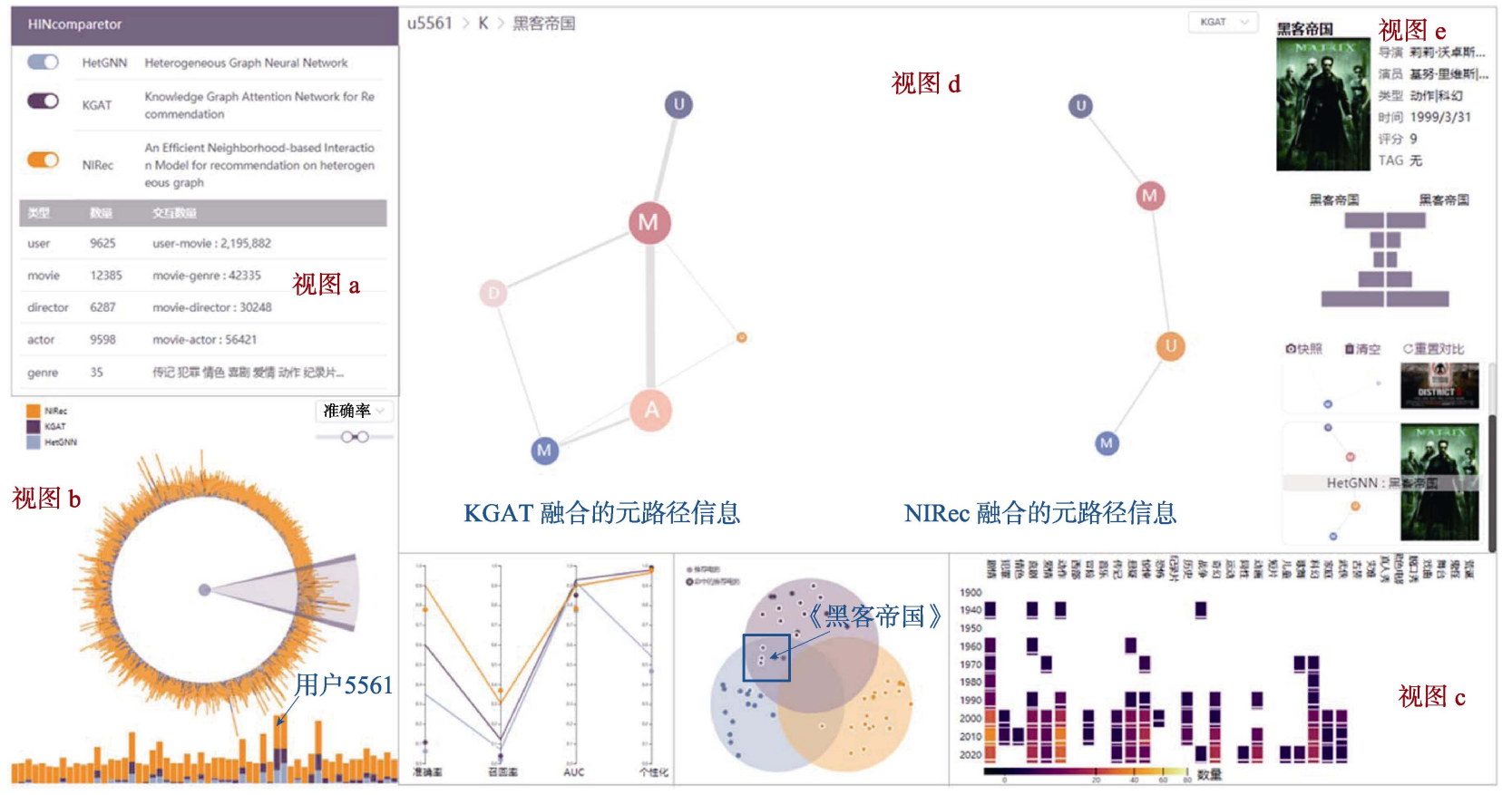

图 3 本文系统结构

照可保存当前推荐结果的路径特征图, 后续可将其 从快照列表中恢复, 并添加至主面板进行对比分析.

\section{4 异构网络嵌入模型的可视化比较系统}

本文设计并实现了如图 3 所示的可视分析系 统 ${ }^{1}$, 并提供了系统演示视频 ${ }^{\circledR}$.

\section{1 评估指标概览图}

根据需求 R1, 评估指标概览图显示多个模型 评估指标结果. 每个堆叠柱状图代表 3 个异构网络 嵌人模型对同一用户的评估指标结果, 每种颜色分 别代表一种模型, 由于环形区域面积有限, 为系统 增加了多角度的数据篮选功能, 用户可以选择感兴 趣的指标, 拖动滑动条查看指标差值在相应区间范 围内的数据, 环形刷选择感兴趣区域, 系统将在环 形区域下方展示指标细节. 针对开发人员通常需要 从指标人手了解模型表现的需求, 该视图不仅提供 了多模型的总体指标概述, 还允许开发人员深人分 析模型对单一用户的表现, 从更加细粒度的评估分 数中探索和分析模型.

\section{2 模型细节评估图}

该视图由平行坐标图、韦恩图和热力图组成. 平行坐标图对评估指标概览图进行补充, 展示了 3 个模型对选定目标在 4 个评估指标下的表现, 纵坐
标上的点分别表示 3 个模型对相应指标的均值, 展 示了当前目标分数与均值的关系. 韦恩图中的散点 代表推荐结果, 散点的白色边框表示其属于预测正 确的电影, 展示了不同嵌人模型的推荐结果之间的 相互关系, 从推荐结果的角度展开模型间的对比, 让用户能够探索不同模型的推荐结果之间的差异或 共同模式. 热力图统计了选定目标的历史观影记录 对于不同年代和不同类型的分布情况，展示了目标 观影偏好, 辅助用户理解和评价推荐结果.

\section{3 推荐结果溯源图}

根据设计需求 $\mathrm{R} 3$ 和 $\mathrm{R} 4$, 本文利用可伸缩的力 导引图展示了选定目标及其推荐结果间的元路径. 这些路径(UMUM, UMGM, UMAM, UMDM)分别 代表与目标用户看过相同电影的人还看了什么电 影、与目标用户看过的电影具有相同类型、与目标 用户看过的电影具有相同演员, 以及与目标用户看 过的电影具有相同导演. 其中, 路径的宽度代表该 路径对最终推荐结果的影响程度, 路径越宽, 代表 推荐这部电影给目标用户的理由是该路径隐含意义 的可能性越大. 节点越大, 代表其所包含的实体节 点越多. 点击感兴趣的节点, 可展开查看该类型节 点中所包含的详细实体信息, 即具体观看了哪部电 影、属于哪一类型、由哪位导演指导等更加细粒度 的信息.

(1) https://mgaia.top/HINcompare

(2) https://mgaia.top/HINcompare/video 


\section{4 上下文信息关联图}

该视图根据需求 $\mathrm{R} 5$ 为推荐结果提供电影的上 下文信息. 为了帮助用户分析推荐结果针对该选定 目标的有效性, 计算了目标用户观影历史与推荐结 果在 4 个属性上的相似性系数以及推荐电影的热度, 以直方图的形式显式地展示了推荐结果与该目标用 户偏好之间的关联. 另外, 为了进一步加强对模型聚 合邻居信息机制的对比, 增加了快照功能, 让用户能 够在推荐结果溯源图中对比 2 个推荐结果的路径重 要性,或同一推荐结果在不同模型中的聚合信息.

\section{5 案例及结果分析}

\section{1 指标概览分析}

开发人员在模型对比方面的第一观感是对评 估指标分数进行比较, 因此, 首先查看 3 个模型在 准确率指标中的表现, 如图 4a 所示.

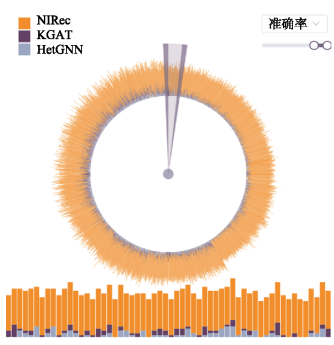

a. 准确率评估

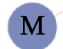

b. NIRec的融合路径
图 4 模型指标概览

从图 4 发现, 与另外 2 个模型相比, NIRec 模型 的评估分数异常高, 即黄色部分占比明显较高, 说明 其预测为正样本的前 20 部电影在测试集中占比很 大. 为此, 随机查看 NIRec 对某一用户的推荐结果, 在其路径关系图中推荐电影与目标用户存在直接相 邻关系, 如图 4b 所示. 随后查看该模型训练过程中 的路径采样结果, 发现这种直接相邻关系在训练过 程中并没有遮盖，而是通过路径间的交互计算进行 了学习, 使该模型能够很轻易地挖掘到用户与电影 之间的交互信息, 并对目标用户推荐该电影, 最终使 在预测精度的评估中表现很好. 因此, 为了对模型进 行更加公平的比较, 针对 NIRec 模型的评估, 不应该 使用预测精度指标，而应使用对正负样本综合分析 的 AUC 指标进行分析. 为了验证这一分析结论, 重 新查看 NIRec 的论文, 发现在其对比实验中使用的 指标是 AUC 和正确率(accuracy, ACC), 未使用准确 率或召回率等常用推荐评估指标, 这一发现证明了 上述分析.
根据以上结论, 重新选择 AUC 指标进行查看, 发现 3 个模型在该指标下的表现差异不大. 为进一 步对比, 使用滑动条对数据进行过滤, 查看指标差 异大于 0.7 的数据. 如图 5a 所示, 该环形堆叠柱状 图的数据很少, 且差异主要集中在分别代表 HetGNN 和 NIRec 的蓝色柱状图和黄色柱状图之间, 而代表 KGAT 模型的紫色柱状图表现较为稳定.

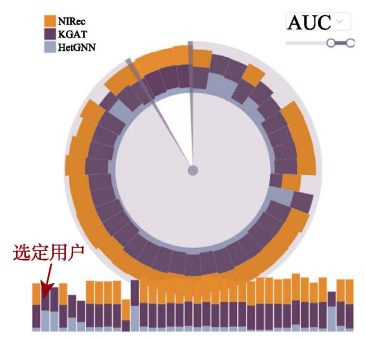

a. AUC指标结果

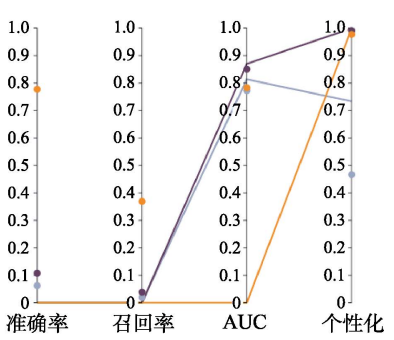

b. 选定用户的指标概览

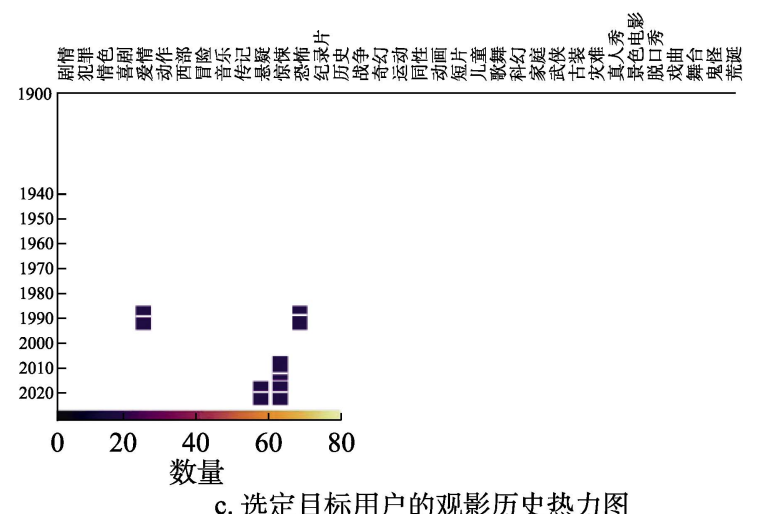

图 5 差异较大的 AUC 指标

接着, 选择图 5a 中红箭头所指的用户, 发现 3 个模型针对该用户的准确率和召回率均为 0 , 且 NIRec 的 AUC 值也为 0 , 如图 $5 b$ 所示.

查看 NIRec 或 HetGNN 模型在 AUC 指标为 0 的其他目标用户发现, 这些用户均存在上述现象, 猜测这种情况是由于数据本身存在问题. 于是, 查 看图 5c 所示用户的观影历史热力图发现, 该目标用 户的观影记录极少, 属于推荐系统中常说的冷启动 问题, 这也就造成模型生成的推荐正样本无法与足 够的测试集进行比较. 这时, 模型在准确率和召回 率的指标下进行评估缺乏意义, 故在进行推荐数据 的清洗时, 常将交互数据小于一定范围的用户数据 删除.

\section{2 推荐结果对比分析}

\section{2 .1 案例 1}

为了探究不同模型的推荐模式, 随机查看了一 些目标用户的推荐结果韦恩图, 发现当准确率明显 高于其均值, 即模型在平行坐标图第 1 栏的值高于 
其对应颜色的点时，韦恩图中代表交集区域的电影 散点数量明显增加, 且目标用户的观影记录热力图 分布较广, 数量较多.

选择用户 5561, 如图 3 中视图 c 所示, 查看其 韦恩图, 发现模型 KGAT 和 HetGNN 的交集区域有 5 部电影, 即蓝色和紫色区域的重合处, 其中有 3 部 属于共同预测成功的电影(命中电影由散点周围白 色的边表示). 随机选择一部电影《黑客帝国》, 查 看推荐该电影的 2 个模型在训练过程中融合的路径 信息, 左边为 KGAT 的融合路径, 右边为 HetGNN 的融合路径, 很明显, 前者所包含的路径信息和连 接关系多于后者. 由此可以看出, KGAT 在网络嵌人 训练过程中学习到了目标用户与推荐结果之间更多 的特征信息

展开较大的演员和电影节点后发现, 所有演员 节点 $A$ 和导演节点 $D$ 均与 2 个电影节点 $M$ 相连, 如图 6 所示, 这 2 部电影分别是《黑客帝国 2: 重装 上阵》和《黑客帝国 3: 矩阵革命》. 由此可见, KGAT 通过传播机制学习到了与历史观看电影相连 接的演员信息和导演信息, 能够融合周围大量的邻 居特征, 保留节点附近复杂的结构特征和丰富的语 义特征，而本文的可视化方法有效地发现并展示了 这种异构网络特征融合机制的特点.

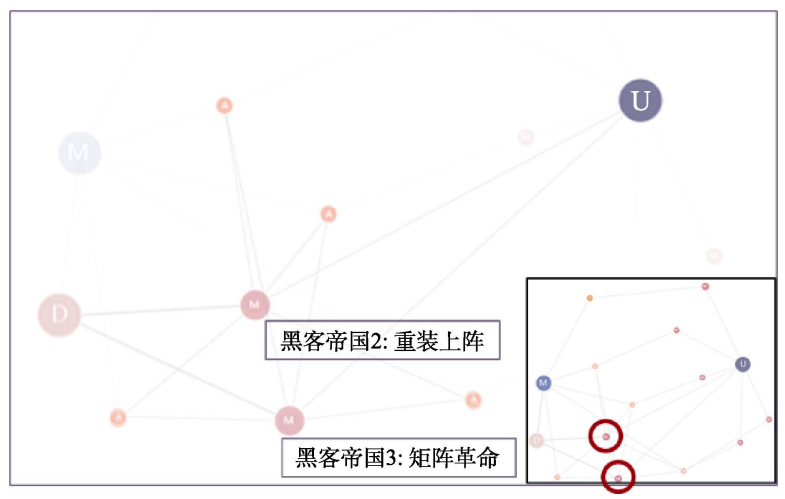

图 6 KGAT 路径溯源图

然而, HetGNN 模型仅融合了看过相同电影的 用户特征(路径 UMUM), 且观察到它在平行坐标中 的个性化评估指标较低, 故猜测该模型总是推荐相 同的电影. 为了验证这种想法, 查看 HetGNN 针对 该目标用户的其他推荐结果发现，它们的特征融合 路径都只有 UMUM, 且在相关性直方图中最后一 项(目标电影的受欢迎程度)的占比总是很高. 如图 7 所示，按其顺序分别代表类型相关性、演员相关 性、导演相关性和标签相关性的属性特征均没有在 HetGNN 的特征融合路径中得到体现.

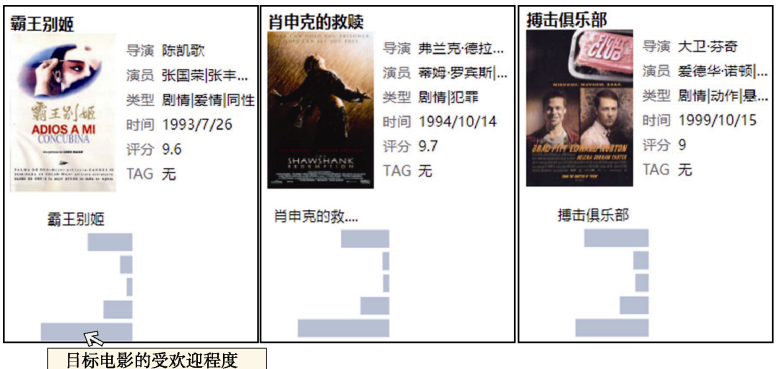

图 7 相关性直方图

\section{2 .2 案例 2}

为了进一步分析在相似指标下不同模型推荐结 果的差异, 将 AUC 指标的差值范围选择为 0.1 , 并随 机选择一位用户(U38), 用户细节信息如图 8 所示.

在图 8a 所示用户指标概览中, NIRec 模型的准 确率指标值为 1.0 , 说明该模型预测为正样本且分 数前 20 的电影均命中了测试集, 而其他模型相差甚 远, 这种关系也可在韦恩图中得以体现.

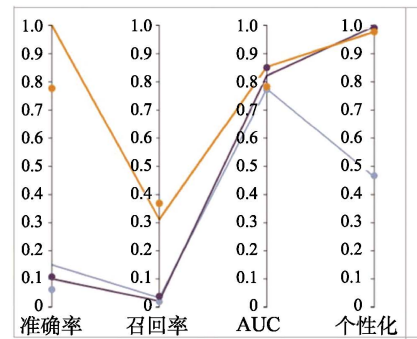

a. 选定用户的指标概览

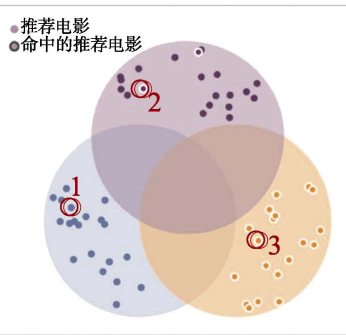

b. 推荐结果分布情况

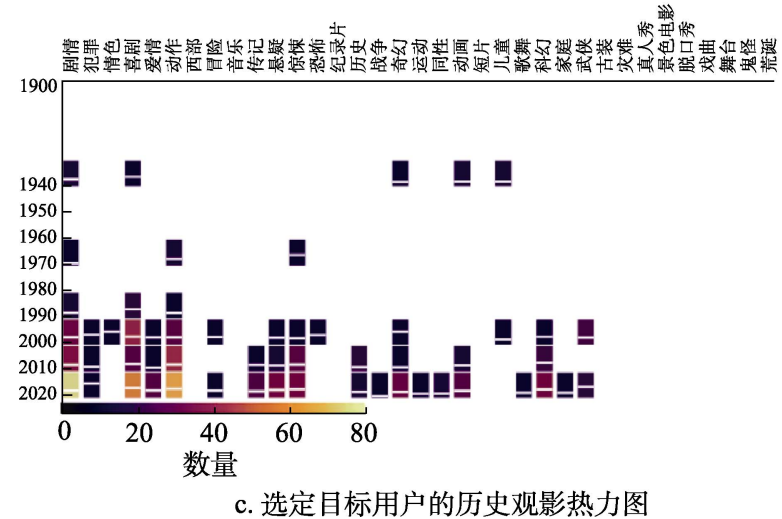

图 8 用户 U38 细节信息展示

接着, 从图 $8 \mathrm{~b}$ 所示 3 个模型的推荐结果中分 别选择了 3 部电影, 其相关性直方图、融合路径信 息和上下文语义信息如图 9 所示. HetGNN 模型推 荐的电影《饮食男女》与用户观影历史的类型相关 性较高, 但在其路径特征中, 目标用户与推荐电影 是 2 个孤点, 并没有挖掘出该用户的邻居特征, 即 个性化特征, 故猜测该电影是根据热门进行推荐的. 因为热门电影的观看用户较多, 连接关系复杂, 其 
融合了周围大量特征信息, 在与其他用户的特征向 量进行点积计算时, 容易得到较高分数, 成为最终 的推荐结果.

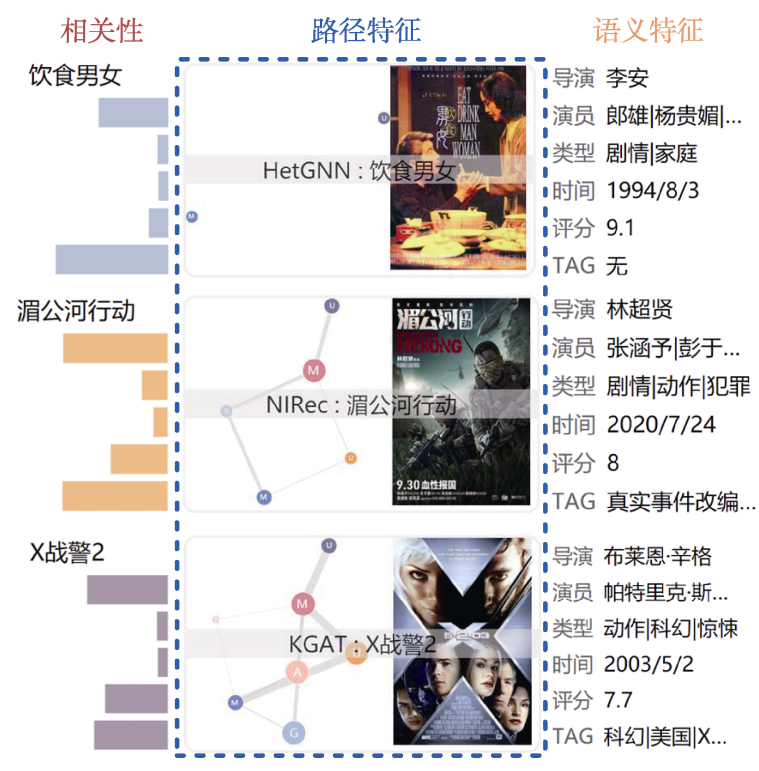

图 9 不同模型对用户 U38 的推荐结果

在 NIRec 和 KGAT 模型的路径特征信息中, 都 包含了元路径 UMUM 和 UMGM. 结合图 8c 进行 综合分析, 可从用户的历史观影热力图中得知, 该 用户非常喜欢“剧情”“动作” “喜剧”类型的电影, 观 看数量大于 40 部, 其次是“悬疑”“惊悚”“奇幻”“科 幻”类电影. 接着查看 NIRec 模型所学习到的路径 特征, 如图 10a 所示展开类型节点 $G$, 包含了 “剧 情”“动作”2 个特征节点, 与上述观察到的用户观影 历史特征相吻合. 而在该电影的类型中还发现了“犯 罪”, 但 NIRec 模型没有学习该特征, 因为用户观看 犯罪类的电影较少，在对目标用户的邻居特征进行 融合时, 学习权重较低, 认为该特征不足以作为用 户的个性化特征进行融合.

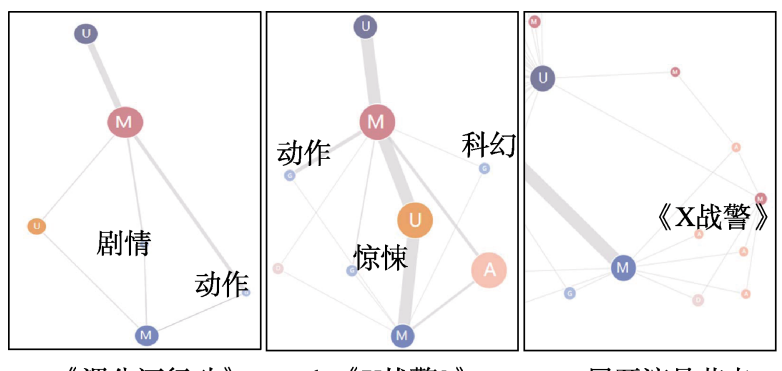

a.《湹公河行动》

b. 《X战警 $2 》$

c. 展开演员节点

图 10 路径特征展开信息

查看 KGAT 模型学习到的路径特征, 并如图 10b 所示展开其类型节点, 包含了 “动作” “惊悚”
“科幻”类电影, 该模型所学类型特征与用户观影历 史特征十分吻合, 与 NIRec 相比, KGAT 还学习到 了元路径 UMAM. 如图 10c 所示展开演员节点 $A$, 该用户观看过电影《X 战警》, 并且电影的演员又 参演了《X 战警 $2 》$, 说明 KGAT 模型学到了异构 图的结构特征, 并将该相似电影推荐给了用户

\section{6 讨 论}

针对 HetGNN 总是推荐相同的电影, 有 2 个疑 惑想要在此讨论: 该模型为什么总是推荐相同的热 门电影? 为什么除了个性化指标外, 该模型的其他 各项评估指标与 KGAT 差异不大?

对于第 1 个问题, 回顾该模型的训练过程, 发 现它利用可重启的随机游走生成邻居集合, 再从集 合中挑选出现频率最高的邻居进行融合. 由于电影 数据集中用户和电影的交互关系(200 万)远远大于 其他类型的边关系 (12 万), 故采样的用户-电影关系 最多, 也就造成该模型总是通过路径 UMUM 推荐 观看人数较多的热门电影. 对于第 2 个问题, 猜想 是由数据本身的长尾性所致, 在电影数据集中, 最 受欢迎的电影通常是大片和经典老片, 这些电影已 为大多数用户所熟知和肯定, 推荐这些电影可能会 使平均评估指标上升, 但对个体而言并非个性化推 荐, 也可能无法帮助用户发现其他新的电影, 但在 冷启动情况下, 这通常被看做一种有效的推荐.

\section{7 结 语}

本文提出的异构网络嵌人模型可视对比分析 系统—HINCompare, 能够对 3 种不同架构的嵌 人模型进行系统性的比较和分析, 可以帮助开发人 员理解不同邻居特征聚合机制对嵌人结果的影响, 并通过案例分析发现了 NIRec 模型的邻居交互学 习问题和 HetGNN 模型的邻居采样融合问题, 证明 了该系统在挖掘模型邻居融合机制方面的有效性. 另外, 推荐结果溯源图能够帮助非模型开发人员快 速理解推荐结果的由来, 以及模型与推荐结果间的 关系, 并利用观影历史热力图和相关性直方图展示 推荐电影的上下文信息和推荐电影与用户偏好之间 的关联, 解决了推荐的黑盒问题, 增加了推荐结果 的可解释性.

未来工作中, 将继续细化针对模型训练过程的 可视信息, 使模型开发人员能够从更细粒度的角度 对模型进行分析, 以便进行更加有效的探索和比较. 


\section{参考文献(References):}

[1] Wang X, Bo D Y, Shi C, et al. A survey on heterogeneous graph embedding: methods, techniques, applications and sources[OL]. [2021-07-06]. https://arxiv.org/pdf/2011.14867.pdf

[2] Shi Chuan, Wang Ruijia, Wang Xiao. Survey of heterogeneous information networks analysis and applications[J/OL]. Journal of Software, 2021: 1-24[2021-07-06]. htt: doi.org/10. 13328/j. cnki.jos.006357(in Chinese)

(石川, 王睿嘉, 王啸. 异质信息网络分析与应用综述 $[\mathrm{J} / \mathrm{OL}]$ 软件学报, 2021: 1-24[2021-07-06]. http://doi.org/10.13328/j. cnki.jos. 006357)

[3] Yuan J, Chen C J, Yang W K, et al. A survey of visual analytics techniques for machine learning[J]. Computational Visual Media, 2021, 7(1): 3-36

[4] Liu M C, Shi J X, Li Z, et al. Towards better analysis of deep convolutional neural networks[J]. IEEE Transactions on Visualization and Computer Graphics, 2017, 23(1): 91-100

[5] Ahn Y, Lin Y R. FairSight: visual analytics for fairness in decision making[J]. IEEE Transactions on Visualization and Computer Graphics, 2020, 26(1): 1086-1095

[6] Zhang C X, Song D J, Huang C, et al. Heterogeneous graph neural network[C] //Proceedings of the 25th ACM SIGKDD International Conference on Knowledge Discovery \& Data Mining. New York: ACM Press, 2019: 793-803

[7] Wang X, He X N, Cao Y X, et al. KGAT: knowledge graph attention network for recommendation[C] //Proceedings of the 25th ACM SIGKDD International Conference on Knowledge Discovery \& Data Mining. New York: ACM Press, 2019: 950958

[8] Jin J R, Qin J R, Fang Y C, et al. An efficient neighborhoodbased interaction model for recommendation on heterogeneous graph[C] //Proceedings of the 26th ACM SIGKDD International Conference on Knowledge Discovery \& Data Mining. New York: ACM Press, 2020: 75-84

[9] Yang C, Xiao Y X, Zhang Y, et al. Heterogeneous network rep- resentation learning: a unified framework with survey and benchmark[J/OL]. IEEE Transactions on Knowledge and Data Engineering, 2020. [2021-07-06]. http://doi.org/10.1109/TKDE. 2020.3045924.

[10] Shi C, Hu B B, Zhao W X, et al. Heterogeneous information network embedding for recommendation[J]. IEEE Transactions on Knowledge and Data Engineering, 2019, 31(2): 357-370

[11] Chen H X, Yin H Z, Wang W Q, et al. PME: projected metric embedding on heterogeneous networks for link prediction[C] //Proceedings of the 24th ACM SIGKDD International Conference on Knowledge Discovery \& Data Mining. New York: ACM Press, 2018: 1177-1186

[12] Wang X, Ji H Y, Shi C, et al. Heterogeneous graph attention network[C] //Proceedings of the World Wide Web Conference. New York: ACM Press, 2019: 2022-2032

[13] Hu Z N, Dong Y X, Wang K S, et al. Heterogeneous graph transformer[C] //Proceedings of the Web Conference 2020. New York: ACM Press, 2020: 2704-2710

[14] Zagoruyko S, Komodakis N. Deepcompare: a study on using convolutional neural networks to compare image patches[J]. Computer Vision and Image Understanding, 2017, 164: 38-55

[15] Ren D H, Amershi S, Lee B, et al. Squares: supporting interactive performance analysis for multiclass classifiers[J]. IEEE Transactions on Visualization and Computer Graphics, 2017, 23(1): 61-70

[16] Cao K L, Liu M C, Su H, et al. Analyzing the noise robustness of deep neural networks[J]. IEEE Transactions on Visualization and Computer Graphics, 2021, 27(7): 3289-3304

[17] Gleicher M, Albers D, Walker R, et al. Visual comparison for information visualization[J]. Information Visualization, 2011, 10(4): 289-309

[18] Zeng H P, Haleem H, Plantaz X, et al. CNNComparator: comparative analytics of convolutional neural networks[OL]. [2021-07-06]. https://arxiv.org/abs/1710.05285v1

[19] Alexander E, Gleicher M. Task-driven comparison of topic models[J]. IEEE Transactions on Visualization and Computer Graphics, 2016, 22(1): 320-329 